\title{
Surgical Results of Posterior Lumbar Interbody Fusion with Transpedicular Fixation in Management of Spondylolisthesis
}

\author{
Ahmed Taha* (D), Mohamed Youssef \\ Neurosurgery Department, Faculty of Medicine, Al-Azhar University, Damietta, Egypt \\ Email:^ahmdth231@gmail.com,youssef200877@yahoo.com
}

How to cite this paper: Taha, A. and Youssef, M. (2020) Surgical Results of Posterior Lumbar Interbody Fusion with Transpedicular Fixation in Management of Spondylolisthesis. Open Journal of Modern Neurosurgery, 10, 146-156.

https://doi.org/10.4236/ojmn.2020.101015

Received: February 20, 2019

Accepted: March 13, 2019

Published: December 31, 2019

Copyright ( 2020 by author(s) and Scientific Research Publishing Inc. This work is licensed under the Creative Commons Attribution International License (CC BY 4.0).

http://creativecommons.org/licenses/by/4.0/

\begin{abstract}
Objective: To evaluate the surgical results of posterior lumber interbody fusion with transpedicular fixation with rod screw system in management of spondylolisthesis. Study Design: Retrospective study reviewed all patient treated by lumber interbody fusion with transpedicular fixation with rod screw system. Patients and Methods: They were 40 patients operated for lumber and lumbosacral spondylolisthesis from Feb 2014 to April 2017 in Al-Azhar university hospital. These patients followed postoperatively clinically for improved neural function and for fusion stability and hardware fixation by radiological investigation. Data about pain intensity (by Visual Analogue Scale) was collected pre- and postoperatively; and outcome was assessed by Oswetry disability index (ODI). Outcome was graded as excellent, good, fair, or poor. Pre- and Post-operative data were statistically compared. Results: The mean age was 45 years (range between 30 - 60 years) with female sex predominance (male:female $=1: 3)$. They had lytic $(n=30)$ or degenerative $(\mathrm{n}=10)$ spondylolisthesis; and all underwent PLIF (posterior lumbar interbody fusion). In lytic group, the level was L4/L5 in 19 patients and L5/S1 in 11 patients, while in degenerative group the level was L4/L5 in 4 patients, L5/S1 in 3 patients, L2/L3 in 2 and L3/L4 in one patient. The spondylolisthesis grade was grade I among 25 subjects, grade two among 11 subjects and retrolisthesis among 4 subjects. Sensory deficits reported in 22 subjects (19 had lytic and 3 had degenerative spondylolisthesis); while motor deficits reported among 10 subjects and reduced reflexes among 8 subjects. The outcome was excellent, good, and fair among 30, 7, and 3 subjects respectively. The return to previous levels of activity was reported among 32 patients. VAS was $3.5 \pm$ 2.94 at the end of follow up, while ODI was $28 \%$ and $36.0 \%$ and 3 and 6 months respectively. Conclusion: PLIF associated with transpedicular rod screw fixation system for management of spondylolisthesis is considered a
\end{abstract}


safe and effective surgical intervention in both lytic and degenerative types.

\section{Keywords}

Spondylolisthesis, Posterior Lumbar Interbody Fusion, Transpedicular Fixation

\section{Introduction}

Spondylolisthesis is defined as the "subluxation of a vertebral body over another in the sagittal plane". It is a frequent mechanism responsible for intervertebral instability [1] [2]. In addition, spondylolisthesis could be attributed to Pars interarticularis defects, previous surgical intervention or trauma leading to laxity of ligaments. In general populations, spondylolisthesis affects about 5.0\% with no specific age predilection [3]. Indications of surgical treatment of spondylolisthesis included claudication of neurogenic origin, severe radicular pain, intractable low back pain, instability, neurological manifestations, failure of medical treatment, progressive course, grades III and IV of listhesis and spondyloptosis [2] [4] [5].

In addition, spondylolisthesis could be due to a spondylolysis. Marchetti and Bartolozzi [6] classification investigates two broad etiological causes (developmental or acquired) (Table 1). The classification is relevant to many disease criteria (e.g., natural history, progression risk, and had treatment implications). On the other side, the Meyerding's classification [7] aims to assess severity of disease using lateral radiographs to judge the potential anterior displacement of the cephalad vertebral body over its distal counterpart.

As previously state, in general population, the incidence of disease is around $5 \%$. However, high prevalence (up to $12 \%$ ), was reported in adolescents who had Scheurman's disease, athletes (weight lifters, football players, and gymnastics), indicating that, the disease could be due to mechanical factors [8].

Congenital predisposition to spondylolysis was proposed in many studies, with prevalence rate of $27 \%-69 \%$ in families of affected members [9]. In addition, the condition was found to be associated with high incidence of spina bifida $(28 \%-42 \%)$, congenital sacrum and superior sacral facet deficiencies [10].

In addition, it is thought that, structural failure in a dysplastic spine could be due to repetitive traumatic stress on the pars inter-articularis [11]. The defects in pars are usually acquired and rarely seen in newborn [8]. L4 and L5 represented the keystones of lumbo-sacral spine as they provide stability by supporting physiological loads and stopping unnecessary motion. Both are the most commonly affected vertebrae. The course of the disease is usually benign as reported in long term follow up longitudinal studies [12]. However, disease progression with neurological affection and low back pain were reported in association with the low sacral index, degenerative disc of Meyerding grade [13].

In patients with isthmic spondylolisthesis, the incidence of spondylolysis may 
be elevated up to $70 \%$ [11]. The progression to spondylolisthesis from spondylolysis is as low as $4 \%-5 \%$ [7]. The risk factors responsible for increased likelihood of further slippage include younger age, female sex, spina bifida, wedging vertebrae, rounded anterior sacral dome and hyperlordosis [14].

Spondylolisthesis could be visualized by standard lateral films, which constitutes the basis for Meyerding's Classification (Figure 1). Oblique radiographs are the best for detection of spondylolysis. The Scottie dog sign of Lachapelle is usually seen on the oblique radiographs as a defect in the collar around the dog's neck. Computed tomography (CT) scan and Single-photon emission computed tomography (SPECT) scans improve recognition (detection) rate of spondylolysis. Magnetic resonance imaging (MRI) scans are used in preoperative period to evaluate neurological compression, surrounding soft tissues and bony anatomy.

When conservative management fails to control pain and/or neurological manifestations, surgical treatment is indicated. Options include neural decompression, bone fusion, and stabilization-fusion with instrumentation.

\section{Aim of the Work}

The aim of this study is to assess the results of surgical intervention after surgery

Table 1. Spondylolisthesis classification proposed by Marchetti and Bartolozzi.

\begin{tabular}{cccc}
\hline \multicolumn{2}{c}{ Developmental } & \multicolumn{2}{c}{ Acquired } \\
\hline High dysplastic & With lysis & Traumatic & Acute fracture \\
& With elongation & & Stress fracture \\
Low dysplastic & With lysis & Post-surgical & Direct \\
& With elongation & & Indirect \\
& & Pathologic & Local \\
& & Systemic \\
& & Degenerative & Primary \\
& & Secondary \\
\hline
\end{tabular}

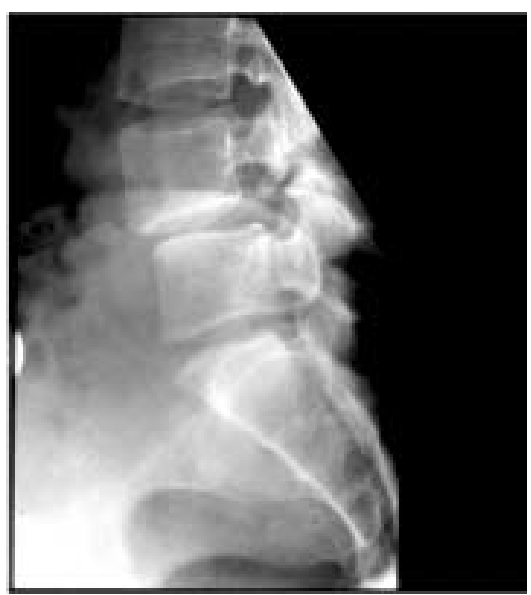

Figure 1. A radiograph showing spondylolisthesis, and Meyerding Grade I spondylolisthesis. 
for spondylolisthesis, using Oswestry Disability Index (ODI) and pain status by Visual Analog Scales (VAS).

\section{Patients and Methods}

Between February 2014 and April 2017, 30 females and 10 males; mean age 45 (rang 30 - 60 years) were submitted to PLIF for treatment of lytic $(\mathrm{n}=30)$ or degenerative $(\mathrm{n}=10)$ spondylolisthesis. They were selected from Al-Azhar University Hospitals (New Damietta). The study was approved by the local institutional review board (IRB), and an informed consent was signed by patients accepting to participate in the study. For inclusion in the study, patients from both genders, with failed conservative treatment for spondylolisthesis were asked to participate. On the other side, patients with spondylolisthesis grade $\mathrm{V}$, those lost regular follow up for 6 months postoperatively, patients with other spinal diseases, patients with previous spine surgery, were excluded from the study.

The level of involvement in patients with lytic spondylolisthesis (30 patients), was L4 - L5 among 19 patients, L5 - S1 among 11 patients. On the other side, the level was L4 - L5, L5 - S1, L2 - L3 and L3 - L4 among 4, 3, 21 patients respectively in degenerative spondylolisthesis. Spondylolisthesis was grade I among 25 patients, grade II among 11 patients and retrolisthesis among 4 patients. Sensory deficits were reported in 22 patients (19 with lytic and 3 with degenerative disease), while motor deficits reported in 10 patients and diminished reflexes among 8 subjects.

Digital X-ray, CT and MRI films of the spine were acquired to measure instability and discover any intraspinal neurological deficits.

Visual analogue score (VAS) [15] for pain and the Oswestry Disability Index (ODI) [16] were implemented for every patient at postoperative 3, 6 and 12 months. Fusion status was radiologically assessed (X-ray, CT or MRI). CT was done when radiological fusion was doubted or proposed to be not achieved.

Outcome was graded by Macnab criteria [17] as excellent (full recovery of symptoms and no limitation of work-related or daily activities), good (residual or occasional symptoms but the subject is able to continue normal daily activities), fair (partial recovery of symptoms, difficulty or inability to work), or poor (no recovery or worsening of symptoms).

Statistical comparison: Collected pre- and postoperative data were statistically compared by the paired $t$ test. On the other side, overall outcome grading was compared by the Chi squared test. A p value of $<0.05$ was considered statistically significant.

\section{Results}

The study included 40 patient ( 30 female and 10 male) diagnosed as spondylolisthesis with failed conservative management. Thus, surgical management was indicated and carried out. All the patients were evaluated by clinical and radiological examinations in pre and postoperative periods (Figures 2-8). 


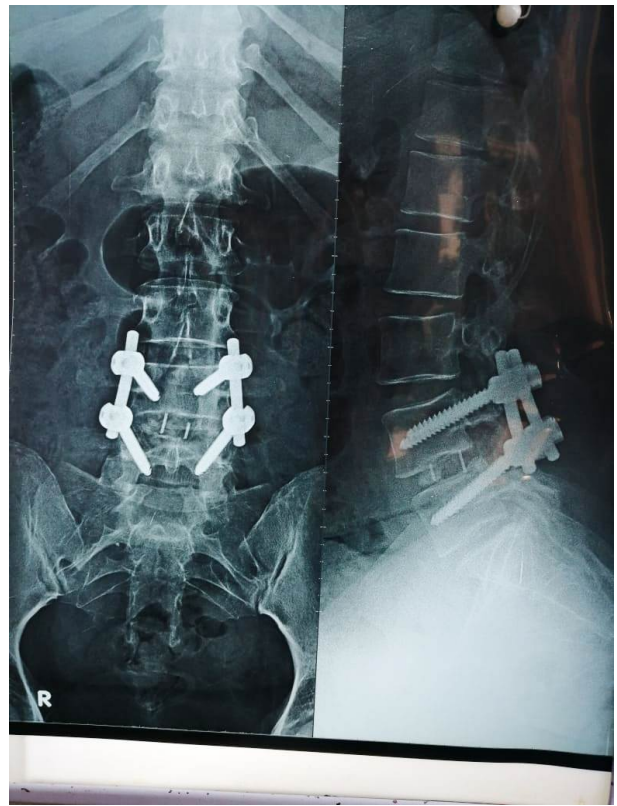

Figure 2. The post-operative X-ray lumbosacral (LS) spine, anterior posterior (AP), and lateral view at 3 months with posterior instrumentation.

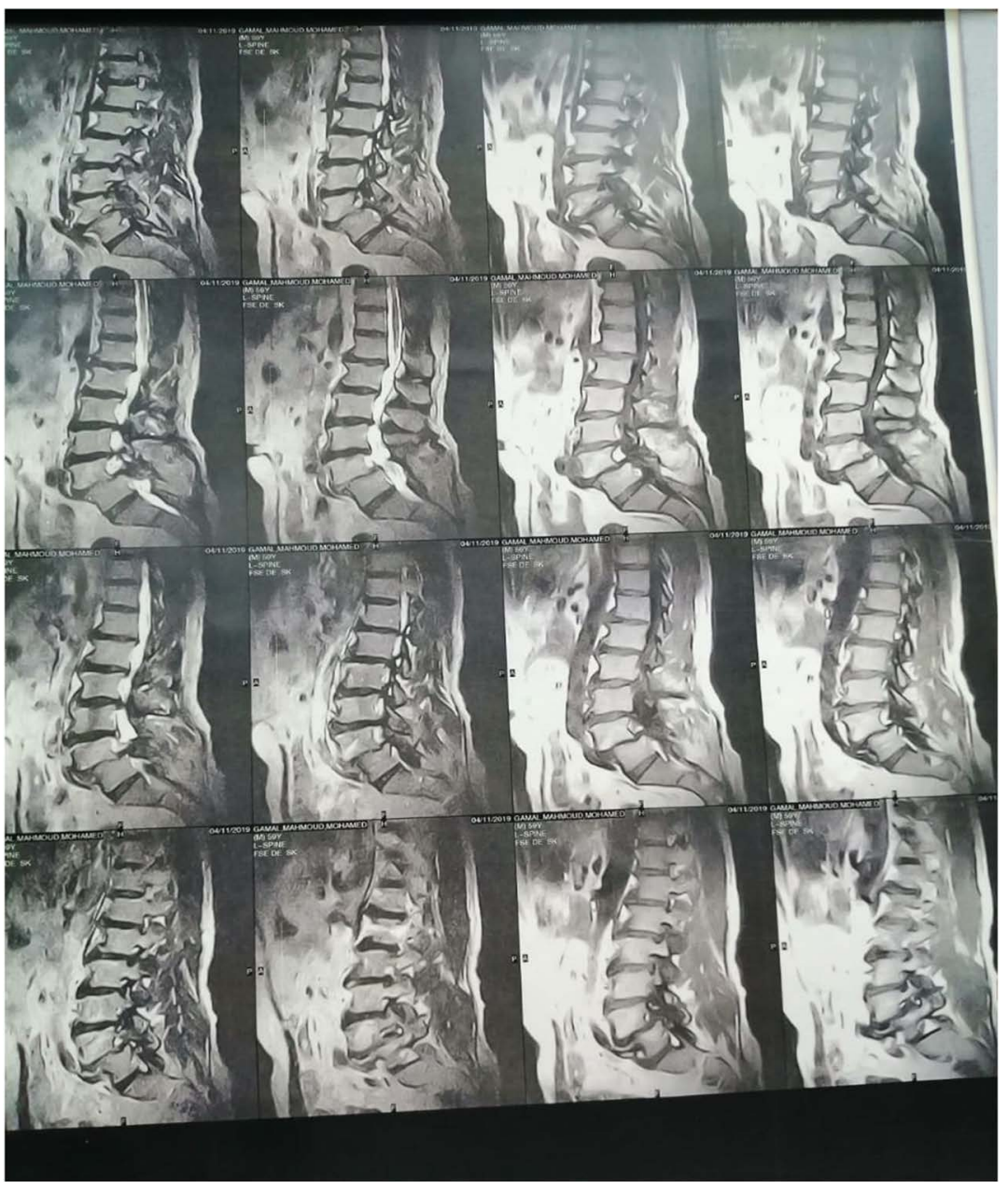

Figure 3. The MRI of lumbo-sacral (LS) spine, sagittal, cut at L4/L5. 


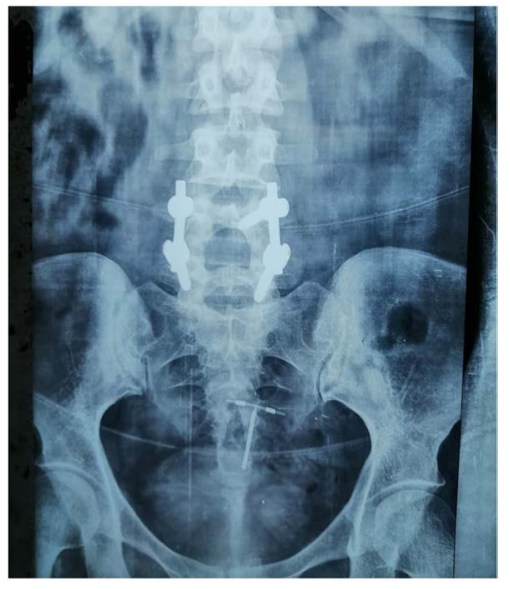

Figure 4. The post-operative X-ray lumbosacral (LS) spine, AP view at 9 months.

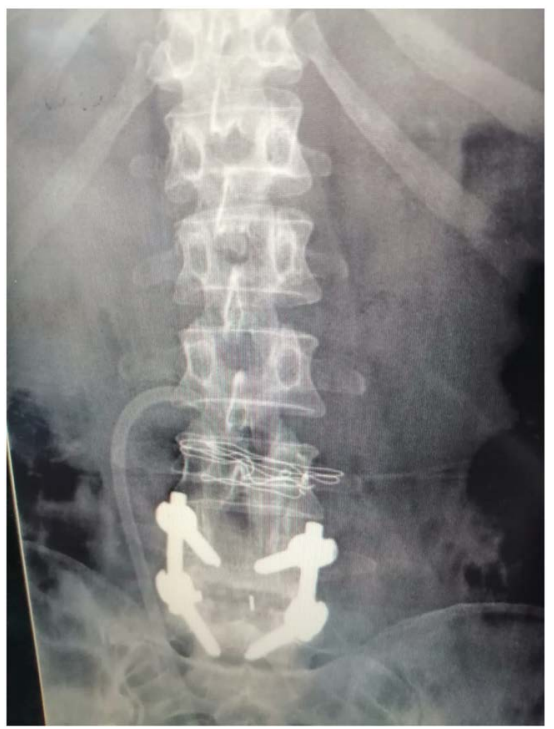

Figure 5. The post-operative X-ray lumbosacral (LS) spine, AP view immediate post-operative.

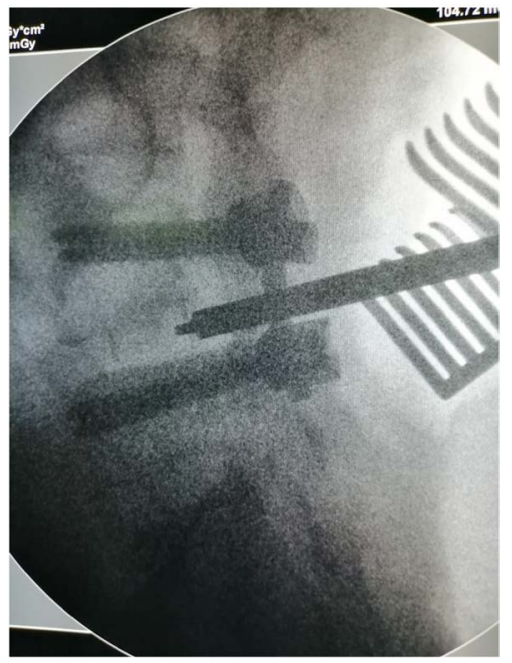

Figure 6. The intraoperative X-ray lumbosacral (LS) spine lateral view. 


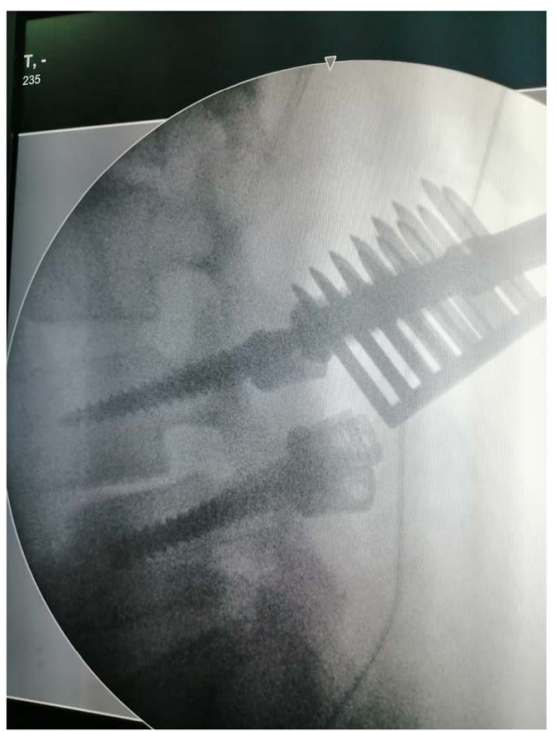

Figure 7. The intraoperative X-ray lumbosacral (LS) spine lateral view.

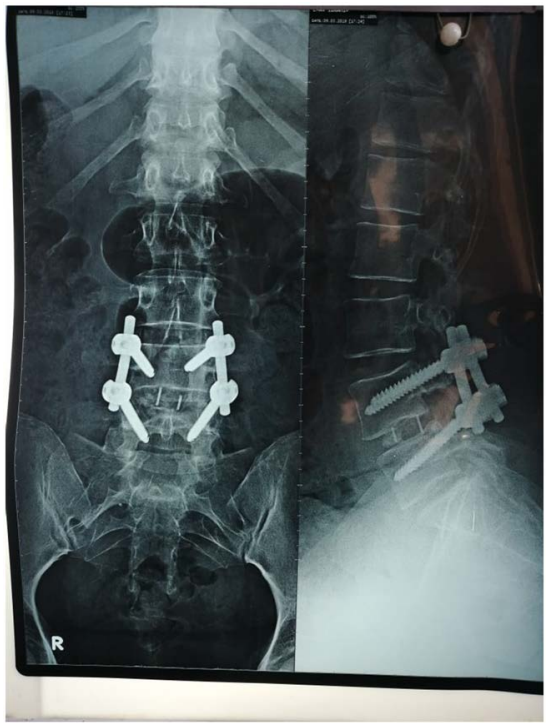

Figure 8. The post-op X-ray LS spine A.P., lateral at 3 months with posterior instrumentation.

The youngest patient included in this series was 30 years, the oldest patient was 60 years with mean age $45 \pm 14.06$ and females were commonly involved (male:female $=1: 3$ ).

All patients complained from low back pain (lumbar pain), reduced range of motion of the lumber area, aggravation of symptoms with prolonged standing. The symptom duration ranged from 1 - 3 years (mean 1.5 years). The most common affected level was L4 - L5, as it was discovered among 19 patients. Instability and its related hypermobility related to the affected level were discovered intraoperatively. The operative time ranged between 45 - 150 minutes (mean value was 100 minutes) and the mean duration of hospital stay was 8.5 days.

The follow up duration was at least 12 months. Revision surgery was reported 
among 3 patients $(7.5 \%)$ due to screw malposition discovered by control CT. No infection or screw break was reported among studied patients. After 6 months postoperative, all patients except 5 subjects had significant improvement in ODI and VAS scores when compared to corresponding preoperative values with $\mathrm{p}$ value $<0.05$. The ODI ranged between 44 and 100, while VAS ranged between 3 and 10; the mean values were 76.78 and 5.78 for preoperative ODI and VAS respectively. Mean postoperative ODI and VAS scores were 27.55 (between 0 - 66) and 2.79 (between 0 - 5), respectively; with statically significant reduction. Five subjects with no resolve of their complaints after the surgery were still heavy workers.

Good outcome was significantly associated with younger age group (31 - 40 years). In addition, good outcome was linked to male gender. Furthermore, good outcome was strongly associated with lower grade of slip, as showed in Table 2.

\section{Discussion}

Surgical management of spondylolisthesis (PLIF) permits decompression of nerves, stabilization of deranged motion segment, restoration of the height of the disc, and restoration of translated sagittal plane and rotational alignment [15]. Age in the present study is comparable to previous studies, reported that, the age ranged from 29.8 to 53.4 years in patients managed for isthmic spondylolisthesis [16] [17]. In another study, the mean age was as that of the present work (45 years) in patients who underwent PLF [18].

Also, the duration of symptoms ranged from 8 to 60 months, with a mean duration of 38.4 months in one study [16], which comparable to the present work.

In our series, level L4-5 involvement was reported in 19 patients, L5 - S1 in 11 subjects and 25 patients had grade 1 slip, while grade II slip was discovered in 11 patients. Kim et al. reported that $50 \%$ of the affected levels were L4-5, and this ratio was similar to that found in the present work.

Posterolateral fusion has been one of the standards surgical interventions for instability of lumbar spine with use of spinal instrumentation. For assessment of

Table 2. Association between outcome and patient age, gender or grade of slips.

\begin{tabular}{cccccc}
\hline & & \multicolumn{3}{c}{ Outcome } & \multirow{2}{*}{ p value } \\
\cline { 3 - 4 } & & Good & Fair & Failed & \\
\hline \multirow{3}{*}{ Age groups } & $41-40(\mathrm{n}=14)$ & 14 & 0 & 0 & \\
& $41-50(\mathrm{n}=7)$ & 4 & 2 & 1 & 0.046 \\
& $51-60(\mathrm{n}=19)$ & 13 & 4 & 2 & \\
Patient gender & Female $(\mathrm{n}=30)$ & 20 & 6 & 4 & 0.038 \\
& Male $(\mathrm{n}=10)$ & 8 & 2 & 0 & \\
Grade of slips & Grade I $(\mathrm{n}=28)$ & 22 & 5 & 1 & 0.022 \\
& Grade II $(\mathrm{n}=12)$ & 7 & 3 & 2 & \\
\hline
\end{tabular}


their outcome, we have used ODI and VAS. They usually used to quantify disability for pain of the lower back, and according to different researchers, this score are considered the gold standard for evaluation of the disability degree and quality of life in low back pain [19]. However, it could be used to estimate the surgical outcome in patients with spinal surgeries.

As in other surgeries, complication can be anticipated, and expected complications in PLIF include CSF leak, infection, failure of implant and neurological deterioration. The long surgery duration could be considered a risk factor for superficial or deep infection of the wound [20]. It is recommended that a decrease in neurological complications may be achieved by the use of intraoperative neuromonitoring especially in surgeries like for spondylolisthesis [21]. In the present work, no implant failure was reported and no mortality was registered. All patients with post-surgical morbidities responded to conservative management.

Some researchers reported that, the clinical outcome for PLIF is not better than other fusion interventions [22]. However, in PLIF, the wider retraction of the nerve root and thecal sac was obviously disadvantageous because it stimulated leg pain. But, an ODI of $89 \%$ with good or excellent results was reported in the PLIF, and $86 \%$ in PLF. The difference was not statistically significant [23] [24].

\section{Conclusion}

In conclusion, surgical decompression and spinal stabilization are advocated for patients who not respond to conservative management and had significant lumbar instability. Careful selection of patients and their clinical patterns govern the choice of surgical intervention from simple decompression to fusion. Instrumented fusion expands the fusion rate. However, complications due to insertions of screws increase in different techniques (i.e., posterior anterior, and or combined approaches have been used for various degree of spondylolisthesis). Posterio-lateral lumbar fusion and transpedicular rod screw systems are effective in the management of spondylolisthesis, as it provided good spinal fusion, less complication with satisfactory surgical outcome.

\section{Conflicts of Interest}

Listed authors declare that, there was no disclosure of interest with any organizations (Financial or non-financial).

\section{References}

[1] Dantas, F., Prandini, M. and Ferreira, M. (2007) Comparison between Posterior Lumbar Fusion with Pedicle Screws and Posterior Lumbar Interbody Fusion with Pedicle Screws in Adult Spondylolisthesis. Arquivos de Neuro-Psiquiatria, 65, 764-770. https://doi.org/10.1590/S0004-282X2007000500006

[2] Dai, L., Jia, L., Yuan, W., Ni, B. and Zhu, H. (2001) Direct Repair of Defect in Lumbar Spondylolysis and Mild Isthmic Spondylolisthesis by Bone Grafting, with or 
without Facet Joint Fusion. European Spine Journal: Official Publication of The European Spine Society, The European Spinal Deformity Society, and The European Section of The Cervical Spine Research Society, 10, 78-83.

[3] Watters III, W.C., Bono, C.M., Gilbert, T.J., Kreiner, D.S., Mazanec, D.J., Shafer, W.O. and North American Spine Society. (2009) An Evidence-Based Clinical Guideline for the Diagnosis and Treatment of Degenerative Lumbar Spondylolithesis. The Spine Journal, 9, 609-614. https://doi.org/10.1016/j.spinee.2009.03.016

[4] Frymoyer, J. and Selby, D. (1985) Segmental Instability: Rationale for Treatment. Spine, 10, 280-286. https://doi.org/10.1097/00007632-198504000-00017

[5] Zdeblick, T. (1993) A Prospective, Randomized Study of Lumbar Fusion. Preliminary Results. Spine, 18, 983-991.

https://doi.org/10.1097/00007632-199306150-00006

[6] Marchetti, P.G. and Bartolozzi, P. (1997) Classification of Spondylolisthesis as a Guideline for Treatment. In: Bridwell, K.H., Dewald, R.L., Hammerberg, K.W., et al., Eds., Textbook of Spinal Surgery, 2nd Edition, Lippincott-Raven, Philadelphia, PA, 1211-1254.

[7] Meyerding, H.W. (1932) Spondylolisthesis. Surgery, Gynecology \& Obstetrics, 54, 371.

[8] Fredrickson, B.E., Baker, D., McHolick, W.J., Yuan, H.A. and Lubicky, J.P. (1984) The Natural History of Spondylolysis and Spondylolisthesis. The Journal of Bone \& Joint Surgery, 66, 699-707. https://doi.org/10.2106/00004623-198466050-00008

[9] Albanese, M. and Pizzutillo, P.D. (1982) Family Study of Spondylolysis and Spondylolisthesis. Journal of Pediatric Orthopaedics, 2, 496-499. https://doi.org/10.1097/01241398-198212000-00006

[10] Wynne-Davies, R. and Scott, J.H. (1979) Inheritance and Spondylolisthesis: A Radiographic Family Survey. The Journal of Bone and Joint Surgery. British Volume, 61B, 301-305. https://doi.org/10.1302/0301-620X.61B3.383720

[11] Wiltse, L.L. and Jackson, D. (1976) Treatment of Spondylolisthesis and Spondylolysis in Children. Clinical Orthopaedics and Related Research, 117, 92-100. https://doi.org/10.1097/00003086-197606000-00012

[12] Saraste, H. (1987) Long Term Clinical and Radiological Follow-up of Spondylolysis and Spondylolisthesis. Journal of Pediatric Orthopaedics, 7, 631-638.

[13] Hammerberg, K.W. (2005) New Concepts on the Pathogenesis and Classification of Spondylolisthesis. Spine, 30, S4-S11. https://doi.org/10.1097/01.brs.0000155576.62159.1c

[14] Lonstein, J.E. (1999) Spondylolisthesis in Children: Cause, Natural History, and Management. Spine, 24, 2640-2652.

https://doi.org/10.1097/00007632-199912150-00011

[15] Spruit, M., Pavlov, P.W., Leitao, J., De Kleuver, M., Anderson, P.G. and Den Boer, F. (2002) Posterior Reduction and Anterior Lumbar Interbodyfusion in Symptomatic Low-Grade Adult Isthmic Spondylolisthesis: Short-Term Radiological and Functional Outcome. European Spine Journal, 11, 428-433.

https://doi.org/10.1007/s00586-002-0400-3

[16] Ganju, A. (2002) Isthmic Spondylolisthesis. Neurosurgical Focus, 13, 1-6. ttps://doi.org/10.3171/foc.2002.13.1.2

[17] Fraser, R.D. (1995) Interbody, Posterior, and Combined Lumbar Fusions. Spine, 20, 167S-177S. https://doi.org/10.1097/00007632-199512151-00016

[18] Jacobs, W.C.H., Vreeling, A. and De Kleuver, M. (2006) Fusion for Low-Grade 
Adult Isthmic Spondylolisthesis: A Systematic Review of the Literature. European Spine Journal, 15, 391-402. https://doi.org/10.1007/s00586-005-1021-4

[19] van Hooff, M.L., Mannion, A.F., Staub, L.P., Ostelo, R.W. and Fairbank, J.C. (2016) Determination of the Oswestry Disability Index Score Equivalent to a "Satisfactory Symptom State" in Patients Undergoing Surgery for Degenerative Disorders of the Lumbar Spine-A Spine Tango Registry-Based Study. The Spine Journal, 16, 1221-1230. https://doi.org/10.1016/j.spinee.2016.06.010

[20] Sasso, R.C. and Garrido, B.J. (2008) Postoperative Spinal Wound Infections. Journal of the American Academy of Orthopaedic Surgeons, 16, 330-337. https://doi.org/10.5435/00124635-200806000-00005

[21] Thuet, E.D., Padberg, A.M., Raynor, B.L., Bridwell, K.H., Riew, K.D., Taylor, B.A. and Lenke, L.G. (2005) Increased Risk of Postoperative Neurologic Deficit for Spinal Surgery Patients with Unobtainable Intraoperative Evoked Potential Data. Spine, 30, 2094-2103. https://doi.org/10.1097/01.brs.0000178845.61747.6a

[22] Ogilvie, J.W. (2005) Complications in Spondylolisthesis Surgery. Spine, 30, S97-101. https://doi.org/10.1097/01.brs.0000155581.81997.80

[23] Hacker, R.J. (1997) Comparison of Interbody Fusion Approaches for Disabling Low Back Pain. Spine, 22, 660-665. https://doi.org/10.1097/00007632-199703150-00017

[24] Wetzel, F.T. and LaRocca, H. (1991) The Failed Posterior Lumbar Interbody Fusion. Spine, 16, 839-845. https://doi.org/10.1097/00007632-199107000-00027 